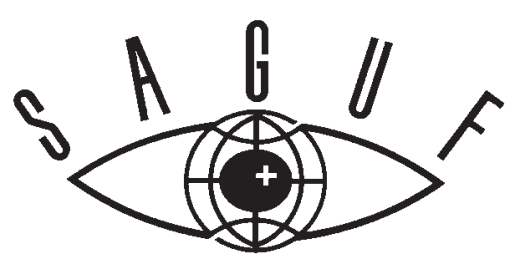

Schweizerische Akademische Gesellschaft für Umweltforschung und Ökologie

Societé Académique Suisse pour la Recherche sur l'Environnement et l'Ecologie

Swiss Academic Society for Environmental Research and Ecology

\section{Nachhaltige Entwicklung erfassen - KLARTEXT spielen}

\author{
Ein neues Kartenspiel kombiniert Spielspaß und Wissensvermittlung: Über \\ Alltagssituationen erfahren Spieler(innen) von „KLARTEXT - Mit Fakten und \\ Fantasie in die Zukunft" Wissenswertes zur
} nachhaltigen Entwicklung in der Schweiz. Dabei lernen sie auch Inhalt und Aufbau des schweizerischen Indikatorensystems zum Monitoring der nachhaltigen Entwicklung (MONET) kennen.
$\mathrm{V}$ erschiedenste Alltagssituationen, über die in den Medien berichtet wird, tragen zum Gesamtbild einer nachhaltigen Entwicklung bei (Jesinghaus 1999). Damit die enthaltenen Informationen in offizielle Systeme von Nachhaltigkeitsindikatoren aufgenommen werden können, müssen sie statistisch verarbeitet werden. Dann nämlich sprechen sie Klartext. Gemäß Born und de Haan (2002, S. 2) sind Nachhaltigkeitsindikatoren „definierbare, messbare Kennwerte, deren absolute Werte bzw. Veränderungsgeschwindigkeit und -richtung aufzeigen sollen, ob sich ein Land (...) im Zeitverlauf in Richtung einer nachhaltigen Entwicklung bewegt“.

Die Schweiz stützt sich auf das Nachhaltigkeitsverständnis der BrundtlandKommission (WCED 1987). Die Bedürfnisse der Menschen sollen befriedigt, dabei aber Gerechtigkeitsaspekte und die Grenzen der Belastbarkeit der natürlichen Umwelt berücksichtigt werden. Diese komplexe Herausforderung wird in der Schweiz mit dem dreidimensionalen Modell der nachhaltigen Entwicklung dargestellt. Danach verfolgt nachhaltige Entwicklung die drei Ziele „gesellschaftliche Solidarität“, „wirtschaftliche Leistungsfähigkeit“ und „ökologische Verantwortung“, zusätzlich konkretisiert mit Postulaten. Diese bilden den Bezugsrahmen, mit dem sich beobachtete Entwicklungen hinsichtlich ihrer Nachhaltigkeitsausrichtung einordnen lassen. Gleichzeitig erlauben die Forderungen eine konsistente und transparente Wahl der Indikatoren (BFS et al. 2003).

\section{Monitoring der nachhaltigen Entwicklung in der Schweiz}

Der Schweizerische Bundesrat begann mit dem Beschluss mehrerer Maßnahmen in der Strategie Nachhaltige Entwicklung 2002, den Verfassungsauftrag zur „Förderung der nachhaltigen Entwicklung " umzusetzen. Damit diese Umsetzung überprüft werden kann, verlangt der Bundesrat „die periodische Produktion von Nachhaltigkeitsindikatoren" (Schweizerischer Bundesrat 2002, S. 34).

Der Bund will mit dem Indikatorensystem für das Monitoring der nachhaltigen Entwicklung in der Schweiz (MONET) Bevölkerung und Entscheidungsträger(innen) regelmäßig über den aktuellen Stand des Prozesses informieren. In der Bundesverwaltung sind daher Grundlagen erarbeitet und ein Indikatorenset entwickelt worden (vergleiche www.monet.admin.ch). Die Daten dieser Indikatoren müssen natürlich regelmäßig aktualisiert und die Ergebnisse transparent gemacht, das heißt verständlich kommuniziert werden. $\mathrm{Zu}$ diesem Zweck wurde eine zielgruppengerechte Informationsplattform eingerichtet (Schweizerischer Bundesrat 2002, ARE und DEZA 2007).

Das Indikatorensystem MONET hilft, folgende Fragen zu beantworten:

- Befindet sich die Schweiz auf dem Weg einer nachhaltigen Entwicklung?

- In welchen Bereichen sind Fortschritte zu verzeichnen?

- Wo besteht Handlungsbedarf?
Bisher sind diese Fakten nur einem kleinen Kreis von Fachleuten bekannt. Doch damit Schweizer(innen) eine lebensbejahende Zukunft mitgestalten können, müssen die in MONET dargstellten Statistiken und Informationen für sie zugänglich gemacht werden. Dies soll nun über publikumswirksame Maßnahmen geschehen.

\section{Wie nachhaltig entwickelt sich die Schweiz?}

In den meisten Lebensbereichen sind Ansätze zur nachhaltigen Entwicklung vorhanden - dies ist eine der wichtigsten Erkenntnisse von MONET. Gleichzeitig sind aber auch gegenläufige Trends festzustellen (vergleiche ARE 2007):

1. In einigen Bereichen wurden Verbesserungen erzielt, die aber durch andere, negative Effekte wieder aufgehoben werden. Ein Beispiel ist die verbesserte Energieeffizienz bei der Produktion von Gütern und Dienstleistungen. Auf-

\section{Kontakt Autoren:}

Dipl. Natw. ETH Vicente Carabias-Hütter kand. Vorstandsmitglied SAGUF | Institut für Nachhaltige Entwicklung | ZHAW Zürcher Hochschule für Angewandte Wissenschaften E-Mail: cahu@zhaw.ch | www.ine.zhaw.ch

Dr. Markus Ulrich | UCS Ulrich Creative Simulations GmbH | E-Mail: markus.ulrich@ucs.ch | www.ucs.ch

Kontakt SAGUF: SAGUF-Geschäftsstelle | Dr. Beatrice Miranda | ETH Zürich D-UWIS/CHN | 8092 Zürich | Schweiz | E-Mail: saguf@env.ethz.ch | http://saguf.scnatweb.ch 
grund des Konsumanstiegs nahm der Gesamtenergieverbrauch dennoch zu (vergleiche ARE et al. 2006).

2. Verbesserungen innerhalb der Schweiz stehen Verschlechterungen auf globaler Ebene gegenüber. Die zunehmenden Außenhandelsverflechtungen der Schweiz und die zunehmende Reisetätigkeit der Schweizer Bevölkerung sind nicht mit einer Erhöhung der Beiträge für die Entwicklungszusammenarbeit verknüpft.

3. Die verhältnismäßig positive Beurteilung der aktuellen Lage der nachhaltigen Entwicklung könnte auf Kosten zukünftiger Generationen zustande gekommen sein: So erfreuen sich heute viele Schweizer(innen) einer hohen Arbeits- und Lebenszufriedenheit, während 17 Prozent der Jugendlichen nicht einmal über grundlegende Lesekompetenzen verfügen und die Jugendarbeitslosigkeit für Schweizer Verhältnisse hoch ist.

Absolut betrachtet ist die Schweiz von einem "nachhaltigen Zustand" noch weit entfernt (ARE 2007, S. 55). Häberli et al. forderten bereits 2002 dringend Lernprozesse, über die eine nachhaltige Entwicklung in einer zukunftsfähigen, offenen Schweiz sowie weltweit verwirklicht werden kann (Häberli et al. 2002).

\section{Bildung für nachhaltige Entwicklung}

Nach der UN-Konferenz über Umwelt und Entwicklung (United Nations Conference on Environment and Development, UNCED) 1992 orientierte sich auch die Umweltbildung neu: Zu den rein ökologischen Fragestellungen traten Fragen nach globaler Gerechtigkeit sowie ökonomischen und sozialen Zusammenhängen im MenschNatur-Verhältnis (vergleiche EDK 2007 und Siemer 2007). Diese Neuorientierung führte zu dem neuen Begriff „Bildung für nachhaltige Entwicklung (BNE)“ (De Haan 2003, S. 34-35).

Ziel dieser BNE ist Gestaltungskompetenz (AG Qualität \& Kompetenzen 2007) für die Mitwirkung an einer nachhaltigen Entwicklung. Dabei sollen vor allem Kompetenzen gefördert werden, die Menschen zu Empathie befähigen, sie kooperationsund aushandlungsfähig sowie mutig für eigenes Handeln auch auf neuen Wegen

Ordnen Sie nachfolgende Alltagssituationen den entsprechenden MONET-Indikatoren zu. Sie finden diese Indikatoren auf www. monet.admin.ch (> Indikatorensystem MONET).

1. Aus dem Umweltbericht der Firma $\mathrm{H} .:$,Wir verbessern laufend unsere Produktion. Den Treibhausgasausstoß $\left(\mathrm{CO}_{2}\right)$ pro verkaufte Einheit konnten wir erneut senken!“

2. Schlagzeile: „Tendenz ungebremst: Der Energieverbrauch für die Produktion von Gütern und Dienstleistungen steigt weiter an."

3. Die Firma S. Medizinaltechnik hat im letzten Jahr ihre Exporte ins Ausland weiter gesteigert.

4. Debatte im Parlament: „Dieser Kredit ist ein Signal zugunsten der Ärmsten dieser Welt!“ Entgegnet Politiker B.: „Machen wir doch erst die Hausaufgaben im eigenen Land!“

5. Gérard (39, geschieden, zwei Kinder): „Pensioniert sein und ohne Stress, das wäre schön.“

6. Das neue Harry-Potter-Buch wurde von Franziska (13) in Rekordzeit verschlungen.

7. Das Schlafzimmer von Reto L. (25) liegt an einer Hauptstraße mit Schwerverkehr.

8. Der Regierungsrat präsentierte gestern sein drittes Sparprogramm mit schmerzhaften Kürzungen.

9. Nach über 120 Jahren ist erstmals wieder ein Bartgeier in freier Wildbahn geschlüpft.

10. Alle freuen sich! Das Unternehmen L. machte 30 Prozent mehr Umsatz. Die Angestellten kriegen einen Bonus, es entstehen neue Arbeitsplätze und die Gemeinde jubelt über höhere Steuereinnahmen.

11. Alice V. (31, alleinerziehend, Teilzeitangestellte im Service, zahlungsunfähig) meldet sich zum ersten Mal auf dem Sozialamt.

12. Weihnachtsmarkt in Nürnberg. Da fliegt Rosa U. (55) jedes Jahr mit ihrer Freundin hin.

Senden Sie die Lösungen bis 31.08.2008 an cahu@zhaw.ch. Unter den richtigen Zusendungen werden bei der Vernissage von KLARTEXT drei Kartenspiele verlost!

und kritisch im Umgang mit ethischen Fragen machen. Wird problemlösendes Verhalten gelernt und als fruchtbar erfahren, kann sich Gestaltungskompetenz entwickeln (Stoltenberg 2006, S. 20-29).

Mit dem Lehrmittel Kompetenzen für die Zukunft - Nachhaltige Entwicklung konkret nähern sich Kyburz-Graber et al. (2006) diesen Schlüsselkomponenten, verbinden sie aber mit konkreten Inhalten, die für die zukünftige Entwicklung bedeutend sind. Dabei gehen sie von den alltäglichen Bedürfnissen der Menschen aus und fragen danach, was es zum Beispiel heißt, gemäß der Leitvorstellung nachhaltiger Entwicklung zu wohnen, zu reisen oder politisch zu handeln.

\section{Spielend nachhaltige Entwicklung erfassen?}

Spielerische Lernformen haben nach Capaul und Ulrich (2003, Ulrich 2003, 2006) großes Potenzial für die BNE. Lässt sich ein so vielschichtiger und offener Veränderungsprozess wie nachhaltige Entwicklung jedoch tatsächlich so weit strukturieren, dass Lernende im Klassenzimmer oder Seminarhotel damit „spielen“ und dabei wesentliche Kompetenzen erwerben können?

Bei der Entwicklung von Plan- und Lernspielen wird deren Komplexität dem Lernziel angepasst. BNE ist nicht auf eine klare Liste von Inhalten reduzierbar, die gelehrt werden sollen. Daher ist eine hohe Komplexität erforderlich, wenn die vielschichtigen Veränderungsprozesse einer nachhaltigen Entwicklung im Zeitraffer erlebt und mitgestaltet oder Indikatoren zur Beurteilung von Nachhaltigkeit erlernt werden sollen. Für das Erfassen grundlegender Systemeigenschaften können dagegen Instrumente mit stark reduzierter Komplexität hilfreicher sein, weil sie diese Eigenschaften prägnanter vermitteln können.

Welche Form eignet sich nun, um das reichhaltige MONET-Wissen spielerisch und fachlich fundiert zugänglich zu machen? Für ein komplexes Spiel, das die Wechselwirkungen der Indikatoren und Teilziele der nachhaltigen Entwicklung berücksichtigt, reicht die statistische Datenbasis nicht aus. Ein statisches, einfach 
strukturiertes Frage-Antwort-Spiel könnte dagegen leicht ins Banale abgleiten. In diesem Spannungsfeld bewegte sich die Spielentwicklung von KLARTEXT.

\section{Thema Nachhaltigkeit in der Zeitungsredaktion}

Spielentwickler(innen), Nachhaltigkeitsfachleute und Grafik-Expert(inn)en arbeiteten von Anfang an eng zusammen (vergleiche Ulrich 2003, Carabias-Hütter und Renner 2005). Sie definierten Lernziele und testeten verschiedenste Spielideen. Als vielversprechend hat sich die folgende erwiesen:

Die Spieler(innen) verkörpern Chefredakteurinnen und Chefredakteure einer Zeitungsredaktion. Laufend treffen - auf Spielkarten dargestellt (Abbildung 1) - Ereignisse und Meldungen aus dem schweizerischen Alltag zu allen Aspekten nachhaltiger Entwicklung ein (siehe Box). Die Spieler(innen) benötigen die Meldungen für ihre Redaktionsarbeit und werden sich daher um sie reißen. Um Artikel schreiben zu können, müssen die Meldungen aber jeweils zum eigenen Ressort passen. In verschiedenen Spielphasen werden News ersteigert und gehandelt sowie Artikel geschrieben. Weitere Ereignisse beeinflussen den Spielverlauf. Wer am Ende des Spiels die meisten und besten Artikel geschrieben hat, steht hoch in der Gunst des Publikums und gewinnt.

Der Zusatztitel „Mit Fakten und Fantasie in die Zukunft" ist Programm für das Kartenspiel KLARTEXT. Man spielt, denkt sich nicht viel dabei und stellt hinterher fest, dass man Aufbau und Inhalt des Indikatorensystems zum Monitoring der nachhaltigen Entwicklung in der Schweiz begriffen hat. Wer Lust und Interesse hat, kann auf der MONET-Website sein Wissen vertiefen.

KLARTEXT ist ohne Internet spielbar. Für Spielvarianten sowie weiter gehende Informationen und aktualisierte Daten ist jedoch ein eigener Bereich auf der Website www.monet.admin.ch reserviert. Er bietet einen leicht verständlichen Zugang zum Kartenspiel und dem Indikatorensystem. Ein Unterrichtsleitfaden soll zudem Lehrpersonen den Einsatz des Spiels erleichtern.

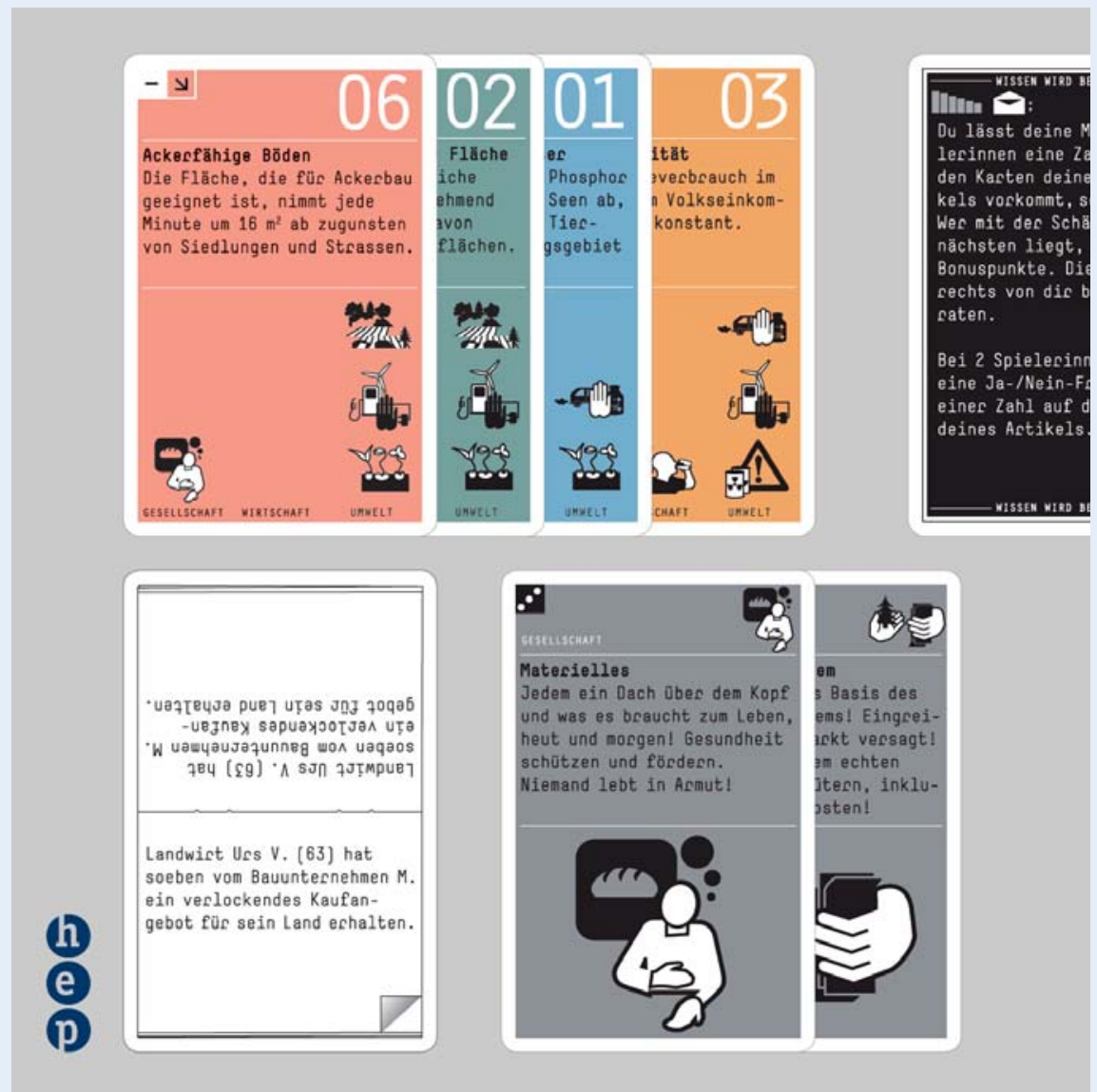

ABBILDUNG 1: Jede Spielkarte entspricht einem Ereignis, einem Indikator des MONET-Systems oder einem der zugrunde liegenden Ziele für die nachhaltige Entwicklung in der Schweiz (Postulate).

Dieses Spiel verbindet Spannung und Spielspaß mit aktuellen Informationen zum Entwicklungsstand der Schweiz in den Bereichen soziale Sicherheit, Gesundheit, Raumnutzung, Kultur, Bildung, Handel, Forschung, Produktion, Konsum, Luft, Abfall, Energie, Artenvielfalt etc. (vergleiche BFS et al. 2003). Damit wird deutlich, wie vielseitig nachhaltige Entwicklung zu verstehen ist, und dass die beispielhaften Alltagssituationen nur einen Ausschnitt aus dieser Themenvielfalt darstellen (zum Beispiel Binswanger 2006). Die spielerische Auseinandersetzung mit diesen Themen und deren Bewertungen hinsichtlich nachhaltiger Entwicklung soll dazu anregen, das eigene Handeln zu überdenken und schließlich selbst Verantwortung zu übernehmen.

\section{Ein ausgezeichnetes Projekt}

Im Sommer 2008 wird das Spiel KLARTEXT beim Lehrmittelverlag h.e.p. als Er- gänzung zum Lehrmittel von Kyburz-Graber et al. (2006) herausgegeben. Damit ist es geeignet für den Schulunterricht, für Weiterbildungsanlässe sowie in der Freizeit „als eine Eingangstüre zur nachhaltigen Entwicklung, welche von der Öffentlichkeit thematisch oft schlecht verstanden oder ignoriert wurde, weil das Thema zu abstrakt und zu weit vom Alltag entfernt erscheint“, so die Schweizerische UNESCOKommission in ihrer Würdigung bei der Projektauszeichnung im Herbst 2007 (Abbildung 2, S. 250).

Nachhaltigkeitsindikatoren sollen zukünftig in aller Munde sein. Daher ist es dringend notwendig, die Kommunikation zur nachhaltigen Entwicklung wirkungsvoll zu gestalten und zu intensivieren (vergleiche ARE und DEZA 2007, Michelsen und Godemann 2005). Dazu sind klare Worte vonnöten: „KLARTEXT - Mit Fakten und Fantasie in die Zukunft" unterstützt dieses Vorhaben. 


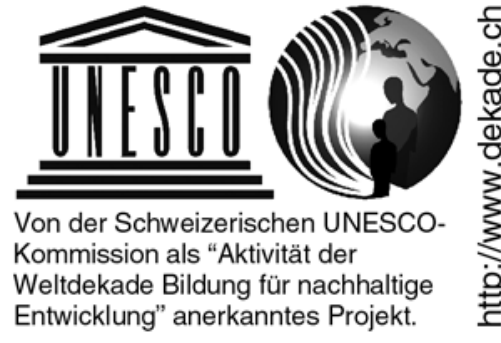

ABBILDUNG 2: Auszeichnung von KLARTEXT durch die UNESCO im Herbst 2007.

\section{Literatur}

AG Qualität \& Kompetenzen. 2007. Orientierungshilfe Bildung für nachhaltige Entwicklung in der Sekundarstufe I. Begründungen, Kompetenzen, Lernangebote. Berlin: Programm Transfer-21. www.transfer-21.de/index.php? $\mathrm{p}=280$ (abgerufen 27.03.2008).

ARE (Bundesamt für Raumentwicklung). 2007. Nachhaltige Entwicklung in der Schweiz Ein Wegweiser. Bern: ARE.

ARE (Bundesamt für Raumentwicklung), DEZA (Direktion für Entwicklung und Zusammenarbeit). 2007. Kommunikation für eine Nachhaltige Entwicklung - Ein Leitfaden. Bern: ARE. ARE (Bundesamt für Raumentwicklung), DEZA (Direktion für Entwicklung und Zusammenarbeit), BAFU (Bundesamt für Umwelt), BFS (Bundesamt für Statistik). 2006. Der ökologische Fussabdruck der Schweiz. Ein Beitrag zur Nachhaltigkeitsdiskussion. Neuchâtel: BFS.

BFS (Bundesamt für Statistik), BUWAL (Bundesamt für Umwelt, Wald und Landschaft), ARE (Bundesamt für Raumentwicklung). 2003. Nachhaltige Entwicklung in der Schweiz. Indikatoren und Kommentare. Neuchâtel: BFS.
Binswanger, M. 2006. Wachstum, nachhaltige Entwicklung und subjektives Wohlempfinden. GAIA 15/1: 69-71.

Born, M., G. de Haan. 2002. Methodik, Entwicklung und Anwendung von Nachhaltigkeitsindikatoren. www.umweltschulen.de/download/ nachhaltigkeitsindikatoren_born_deHaan.pdf (abgerufen 27.03.2008)

Capaul, R., M. Ulrich. 2003. Planpiele - Simulationsspiele für Unterricht und Training. Mit Kurztheorie: Simulations- und Planspielmethodik. Altstätten, $\mathrm{CH}$ : Tobler.

Carabias-Hütter, V., E. Renner. 2005. Indikatoren. Nachhaltige Regionalentwicklung verstehen, messen, bewerten und steuern. Projekt FUNalpin - Arbeitsbericht 5. St. Gallen: Forschungsstelle für Wirtschaftsgeographie und Raumordnungspolitik - Hochschule St. Gallen (FWR-HSG)

De Haan, G. 2003. Bildung für nachhaltige Entwicklung. In: Öko-Lexikon. Herausgegeben von U. Simonis. München: C. H. Beck.

EDK (Schweizerische Konferenz der kantonalen Erziehungsdirektoren). 2007. Massnahmenplan Bildung für Nachhaltige Entwicklung 2007-2014. Bern: EDK.

Häberli, R., R. Gessler, W. Grossenbacher-Mansuy, D. Lehmann Pollheimer. 2002. Vision Lebensqualität. Nachhaltige Entwicklung - ökologisch notwendig, wirtschaftlich klug, gesellschaftlich möglich. Synthesebericht des Schwerpunktprogramms Umwelt Schweiz. Zürich: vdf.

Jesinghaus, J. 1999. Indicators for decision-making. Ispra: European Commission, Joint Research Centre of the European Commission (JRC), (The European Commission's) Institute for Systems, Informatics and Safety (ISIS).

Kyburz-Graber, R. et al. 2006. Kompetenzen für die Zukunft - Nachhaltige Entwicklung konkret. Bern: h.e.p.

Michelsen, G., J. Godemann. 2005. Handbuch Nachhaltigkeitskommunikation. Grundlagen und Praxis. München: oekom.
Schweizerischer Bundesrat. 2002. Strategie Nachhaltige Entwicklung 2002. Bericht vom 27. März 2002. Bern: Bundesamt für Raumentwicklung (ARE).

Siemer, S. H. 2007. Das Programm der Bildung für nachhaltige Entwicklung. Eine systematische Diagnose mit den Schemata Qualität und Nach haltigkeit. Dissertation, Leuphana Universität Lüneburg.

Stoltenberg, U. 2006. Chancen des Konzepts der Bildung für nachhaltige Entwicklung für die Kooperation von Schule und außerschulischer (Umwelt) Bildung. In: Fit in die Zukunft. Herausgegeben von M. Loewenfeld, S. Kreuzinger. München: oekom.

Ulrich, M. 2003. Mit Planspielen Nachhaltige Entwicklung erleben! Der Beitrag der Planspielmethodik zur Bildung über Nachhaltigkeit. DGU Nachrichten 27.

Ulrich, M. 2006. Komplexität anpacken: Mit Planspielen erfolgreiches Handeln erlernen. In: Komplexität erkennen - Zukunft gestalten. Bad Soden: Werner-und-Elisabeth-Kollath-Stiftung. WCED (World Commission on Environment and Development). 1987. Our Common Future. Oxford: WCED.

\section{Weiterbildungsangebot}

Die Autoren stellen KLARTEXT gerne persönlich vor, etwa bei Fortbildungskursen für Lehrende oder in Unternehmen. Das Kursangebot reicht von „Nachhaltige Entwicklung spielerisch vermitteln" über Bildungsveranstaltungen mit Plan- und Simulationsspielen bis hin zu Nachhaltigkeitsmanagement in Unternehmen.

WEITERE INFORMATIONEN: Vicente Carabias-Hütter/Markus Ulrich, E-Mail: cahu@zhaw.ch/info@ucs.ch

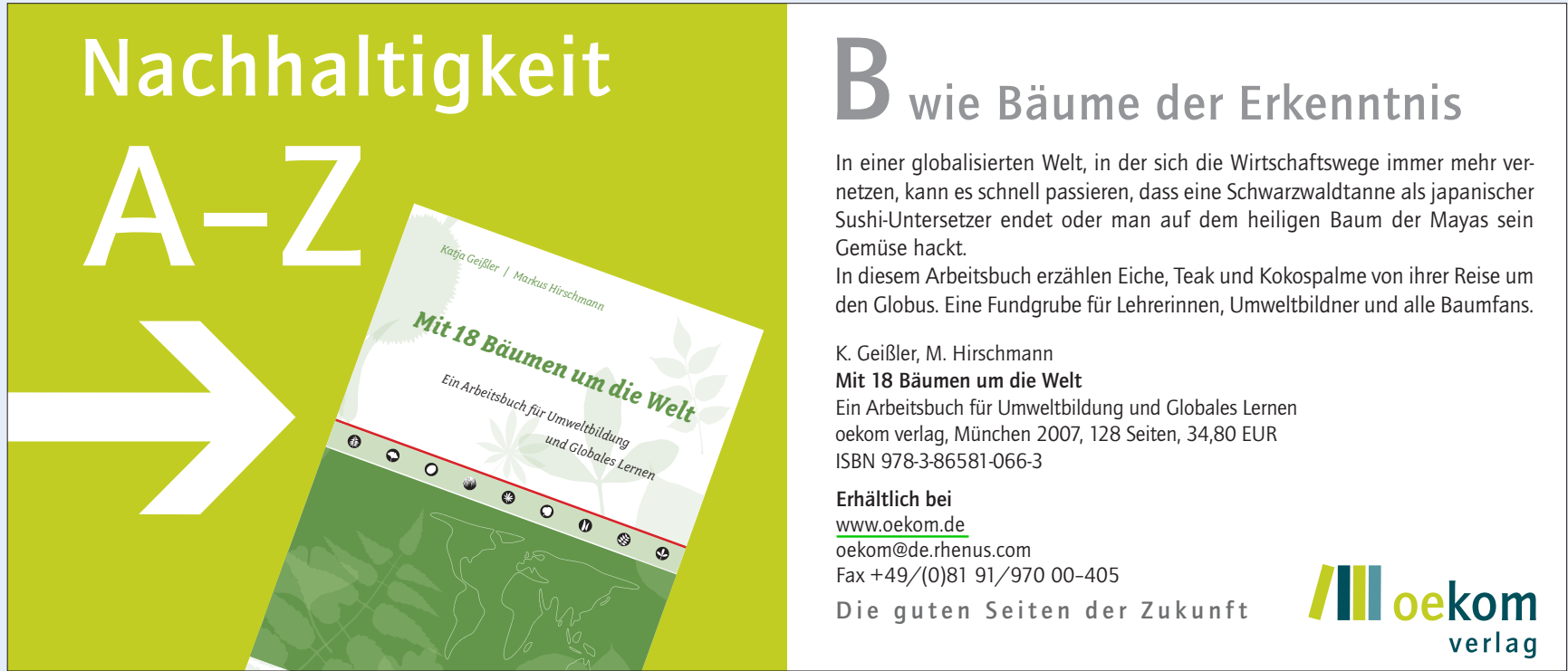

